\title{
Research on the Practice of Health Service for the Elderly Based on the View of Climate
}

\author{
Jixing Gong ${ }^{1}$, Zhiying Lv ${ }^{1}$ \\ ${ }^{1}$ Chengdu University of Information Technology, School of Management, Chengdu, Sichuan, 610203
}

Keywords: health service; the elderly; climate condition

\begin{abstract}
With the progress of the times, the health problems of the elderly have become the focus of society. Attention to the issue of elderly people's health and active aging in their later years is increasingly valued by people, including social work in the health of the elderly, and their professionalism and scientific nature.
\end{abstract}

\section{Introduction}

The World Health Organization uses 60 years as the starting age of the elderly. The reason why the starting point for the elderly started from the age of 60 is because after this age, people's various physiological functions have undergone major degenerative changes, most of which have shown a decline and decline. After entering the 1990s, developing countries have also experienced the historical process of population aging. At present, the growth rate of the elderly population in developing countries is $3.1 \%$, which is three times that of developed countries; the number of elderly people in the world has increased by 1.2 million each month. People, of which developing countries account for more than $80 \%$. It can be said that not only for developing countries, developed countries are also facing the problem of the elderly, which also poses a huge challenge to the relevant policies and social work formulated by various countries. 1 In the establishment of the elderly service system, community service for the elderly can effectively solve the problem of care for the elderly. Now many children in their families have chosen to leave their hometowns and go out to work. This has led to the phenomenon of two elderly people and even one elderly person living alone at home. Some old people are unable to afford high-priced services or enter their own home because of family conditions. All kinds of welfare homes, if they do not go out and enrich their lives, will lead to life in old age facing various difficulties. This not only reduces the quality of life of elderly people in their later years, but also is not conducive to the harmony and stability of the entire society.

\section{The Theoretical Significance of Health Preservation for the Elderly}

“Active Ageing” and "Healthy Ageing” are two slogans on aging that were proposed at the end of the 20th century. The goal of both is the longevity and health of the majority of the elderly population, and the elderly are encouraged to step out of their homes and continue to participate in society. All kinds of activities and cultures in China make it possible to radiate an active and healthy style from the inside out. According to the relevant literature and studies reviewed by the author, there are few examples of the current involvement of social work in the field of healthy living of the elderly in China, and there is no activity that integrates active and healthy aging. It can be said that this is a new kind of academic thinking for social work. Therefore, from the author's point of view, if the theory is based on active and healthy aging, social work is involved in the health of the elderly and the slogan of health, participation, and protection for the elderly is advocated, and more senior citizens are invited to participate in helping the elderly. To increase their enthusiasm, this will be a path worthy of promotion [1]. This article is based on practical exploration and has formed the operating model of the "Hangle Yangshengtang" group, and has put active and healthy aging through it, thereby advancing the use of social support theory and the perspective of superiority and the depth of related academic research. Through participation in group activities, many elderly 
people in the community have a clearer understanding of health and well-being, and they are trying to use health and wellness knowledge in their lives. They also know how to find a scientific solution to their existing health problems. This kind of good momentum of development will greatly promote the operation mode of the "Kangleyue Yangshengtang" group in the future, and it will help improve the quality of life of the elderly in their later years.

\section{The Necessity and Feasibility of Health Maintenance for the Elderly}

With the constant development of Chinese society, people's lives are getting better and better, but there are also many hidden problems that are health problems. Rich dishes make many people suffer from diseases such as high blood fat and high blood pressure, which brings people a lot of troubles. The emergence of these problems has also enabled major TV stations to launch programmes on health and wellness to help people establish a correct outlook on health. Since the establishment of the X Community Service Center, it has carried out many activities for the middle-aged and elderly people in the community to benefit their physical and mental development. They have also organized free medical examinations for them. These activities have been well received by the middle and old age groups. Now, in order to better serve the residents and to promote social development, the community service center believes it is necessary to carry out group activities on health lectures [2].

Most of the service groups in community service centers are middle-aged and senior citizens and children in and around the community, and there are many activities for middle-aged and elderly people. In other words, the $\mathrm{X}$ community service center focuses on the middle-aged and older people. As far as the service center is concerned, the activities that have been carried out include Tai Chi Chuan, Ba Duan Jin, an old dance band, an athletic mahjong, and a choir. Since the launch of these activities, many old friends have been very motivated and very serious in learning. It can be said that these activities have also been recognized by them. However, in terms of practicality, these activities only teach how middle-aged and older people can exercise and enrich their daily lives. They also have questions about what to look for in terms of diet, rest, etc. X Community Service Center did not find a suitable way and way to help them solve these problems. Therefore, the lecture is a good channel for communication and learning. It is also very close to the daily life of the residents. Everyone can intuitively and clearly understand their own health problems and find a correct solution to the problem.

Expanding from case service to group activities is a necessary condition for the "Leisure and Wellness" group. When I first approached the case client, I only solved the problem for her, as she always did [3]. After learning about it later, I knew that the case was over 70 years old. The Shanghai native was a doctor and she worked in Shenzhen. Son lived together. As the investigators usually participate in many of our activities and are actively volunteering, they also know more about the time we normally carry out activities. By chance, during the conversation, the workers of the X community service center learned that the owner of the house had all the discs of the Beijing Yangshengtang program. The case has also been mentioned many times by the fact that due to the unfamiliarity of life, people rarely communicate with others and they usually watch more health programs. The workers then discussed with the owner whether they are willing to come up with their own set of dishes and help us to carry out the group activities of "Health and Wellness Center". And in order to help the case solve her own problems as soon as possible, the workers put forward volunteer doctors who hope that she can come to this group activity. The case was readily agreed upon by the case and we hope that we will start this activity as soon as possible. This not only enriched her leisure activities, but also provided more professional answers to other residents. Of course, the most important thing is to let the owner think that he is useful and he is valuable.

As mentioned above, although the $\mathrm{X}$ community service center has carried out many activities in the elderly, the issues related to the health of the elderly have not been developed before. Moreover, after investigations by social workers at the $\mathrm{X}$ community service center, the surrounding service centers did not personally participate in this type of activity. It can be said that it is a challenge for both the client and the service center to be contacted for the first time. If community service centers 
can participate, not only can they provide more professional and caring services to the elderly, they can also accelerate the rapid development of the group, and they also create a precedent and set an example for the surrounding community service centers. Once successful, they will attract More community service centers will follow suit to find suitable solutions to the health problems of more elderly people [4].

According to our survey, although most of the older people in the community are familiar with each other, if they are allowed to organize this activity spontaneously, it will cause inconvenience in many aspects. For example, the traditional thinking and concept of the elderly, only a small number of people can quickly integrate into them, most of them are still relatively passive. Moreover, the age restrictions make it difficult for them to integrate into the control of modern society. They do not know how to find a suitable health talk program and do not know how to use electronic products to help them achieve their goals. Nor can they find the venue for the team to perform. And these issues will become simple and easy with the participation of social workers. From the professional point of view, the social workers use a series of methods and techniques to help them solve the problems that have arisen in the early stage and the development of the group so as to help themselves.

\section{Countermeasures and Suggestions}

On the basis of unified understanding and clear goals, relevant departments and organizations should incorporate old-age health care into their agendas, combine their own work responsibilities and tasks, and actively advance old-age health work [2]. The streets and communities fully demonstrate their role as the main body, actively demonstrate the functions of the community health service organizations, carry out the health education work on the awareness of healthy health, and improve and adjust the missionary methods to modernize the multimedia means and networks. Ways to make full use of it, to provide health and health knowledge to residents, the information is more authoritative and credible. Healthy health education is not only the exchange of health knowledge, but also plays an important role in the effectiveness and applicability of the formation of healthy health behaviors [4].

Health and wellness service project community can actively carry out. The community old-age community has certain health knowledge and attitudes that can be learned through the survey results. This shows that the concept of healthy health is still believed and accepted by older groups. If certain health-care projects are deployed in the community, old-age groups can be healthy and healthy. Active participation and experience in practical activities is conducive to the formation of healthy behaviors among the elderly population [5]. It is suggested that relevant schools provide a platform for the study of Chinese medicine practitioners, practitioners and pharmacy students. Students of traditional Chinese medicine and related majors go to the community from hospital hospitals to assist and participate in health education and healthy health work, so as to enhance the professionalism of Chinese medical education in the community.

Promote the scientific consciousness of health preservation of the elderly population. Strengthening the construction of hardware on the surface is part of the health science of the elderly, and it is equally important to raise the awareness of the elderly population. In accordance with other relevant policies and regulations and the corresponding venue facilities, various forms of publicity, such as television, newspapers, periodicals, magazines, and radio, should be used to actively carry out propaganda work for the health of the elderly, popularize science knowledge, and advocate lifestyles. Science and health, so that general health knowledge and correct health methods are commonly understood among the elderly, so that the sense of "self-preservation" among the elderly groups is established, so that culture and entertainment are integrated with old-age health activities, and the combination with health care is increasing. The unscientific ways of health care can be corrected so that the awareness of scientific health can be effectively established in the elderly health care group and guide them to scientific health. 


\section{Conclusion}

Allow the elderly groups to properly cultivate health awareness. The consciousness of self-health and health preservation of the current ageing group should be actively promoted through various media and modern propaganda means. The establishment of the concept of health care and consumption should be positive, and the formation of health care consumption habits should be rational. Due to the existence of different conditions, such as differences in occupation, character traits, education level, and living environment, the old people will also show multi-level health care and consumption needs, and they are constantly changing. To properly adjust the industrial structure of the health products industry, not only to produce high-quality health tangible physical products, but also to produce high-quality health intangible products to meet the different levels of health consumption needs.

\section{References}

[1] Shi Xiaoke, A Study of the Quality of Life of Older Adults in Guangzhou City and Its Influencing Factors [J]. Graduate School of Sun Yat-sen University, 2011, 32(3):43-59.

[2] Yan Jing. What kind of challenges will we face in the study of geriatric diseases? [J]. Chinese Journal of Geriatrics Research, 2014, 1(1): 3-5.

[3] Li Chenglai, Zhang Wei, Wang Xin. Study on the Growth Pattern of the Aged Community in the View of Cultural Ecology [J]. Architectural Journal, 2014, 60(11): 96-99.

[4] Li Lei, Qin Wenzhe, Xu Yan, et al. Multi-dimensional Health Status of the Elderly in a Community in Chengdu and Its Influencing Factors [J]. Journal of Preventive Medicine Information, 2016, 32(10): 1027-1033.

[5] Wang Yiming, Chen Hu. China's popular science problems and suggestions for entering an aging society [J]. China Development, 2016, 16(2): 74-79. 